\title{
Inconsistencies in bioelectrical impedance and anthropometric measurements of fat mass in a field study of prepubertal children
}

\author{
Mareike Mast, A. Sönnichsen, K. Langnäse, K. Labitzke, U. Bruse, U. Preuß and M. J. Müller* \\ Institute of Human Nutrition and Food Sciences, University of Kiel, Düsternbrooker Weg 17, D-24105 Kiel, Germany
}

(Received 6 March 2001 - Revised 23 July 2001 - Accepted 5 October 2001)

\begin{abstract}
The present study examined the consistency of bioelectrical impedance analysis (BIA) and anthropometric measurements in body composition analysis in a field study of prepubertal children using a representative group of 2286 5-7-year-old children from Kiel, north-west Germany. Body composition was assessed using anthropometric measures (A; four skinfolds) and BIA. Various published algorithms (according to Lohmann (1986) and Deurenberg et al. (1990) for A, Kushner et al. (1992), Schaefer et al. (1994) and Wabitsch et al. (1996) for BIA and Goran et al. (1996) for a combined approach) were used to estimate body composition. Using A resulted in a sum of four skinfolds varying between age-dependent median values of 24.0 and $28.2 \mathrm{~mm}$ in boys and 30.5 and $33.3 \mathrm{~mm}$ in girls. When fat mass (FM) was calculated from A, age- and algorithm-dependent differences in median values were observed, with values varying between 8.5 and $14.6 \%$ for boys and 11.1 and $14.9 \%$ for girls. Using different algorithms (Lohmann (1986) v. Deurenberg et al. (1990)) only minor inconsistencies were observed. BIA-derived resistance index (height ${ }^{2} /$ resistance) varied between 18.8 and $24.4 \mathrm{~cm}^{2} / \Omega$ for boys and 17.1 and $19.0 \mathrm{~cm}^{2} / \Omega$ for girls. Using four different algorithms to estimate FM from BIA data resulted in high intra-individual variances in percentage FM (from 13.8 to 33.4 ) as well as in the prevalence of overweight (from 14.7 to $98.4 \%$ for boys and from 42.3 to $98.5 \%$ for girls). Data obtained using the different BIA algorithms showed some, or even marked, inconsistencies as well as systematic deviations (an overestimation of FM at low percentage FM, Schaefer et al. (1994) v. Wabitsch et al. (1996)). When comparing BIA with A, BIA systematically overestimated FM. The differences between the results were influenced by BMI, gender and height. Considerable inconsistencies were observed at low BMI $(<10$ th percentile) for girls and for small children. Although the within-observer as well as betweenobserver $\mathrm{CV}$ for both techniques are acceptable, we recommend caution in relation to the algorithms used for data analysis. The use of an interchange table of percentage FM derived from different algorithms for different percentile groups of skinfold thicknesses is recommended.
\end{abstract}

Fat mass: Anthropometry: Bioelectrical impedance: Overweight: Obesity: Children

There is no uniform definition and assessment for overweight and obesity in children. The validity of BMI as an index of fatness has been examined in children (Rolland-Cachera et al. 1991; Dietz, 1994; Cole et al. 2000), but its accuracy has been questioned (Robinson, 1993). In addition, the cut off-points varied considerably. Available data allow neither a meaningful international estimate of the prevalence of obesity nor international comparisons of data (Guillaume, 1999). In view of these limitations it has been suggested that assessment of nutritional state should also include an estimate of body fat (Schaefer et al. 1998).

There are different methods for assessing body composition in children: densitometry, $2 \mathrm{H}$ dilution, total body K, dual-energy X-ray absorptiometry (DXA), anthropometry and bioelectrical impedance analysis (BIA; Lohmann, 1986). Some of these methods are expensive, technically awkward and impractical for use in a field study. Skinfold measurements as well as BIA have been recommended for the assessment of fat mass (FM) in

\footnotetext{
Abbreviations: BIA, bioelectrical impedance analysis; DXA, dual-energy X-ray absorptiometry; FFM, fat-free mass; FM, fat mass; \% FM, percentage fat mass; KOPS, Kiel Obesity Prevention Study.

* Corresponding author: Professor Dr Manfred J. Mueller, fax +49 4318803 6798, email mmueller@ nutrfoodsc.uni-kiel.de
} 
large populations of children (Cordain et al. 1988; Houtkooper et al. 1989; Kushner et al. 1992; Schaefer et al. 1994; Goran et al. 1996; Wabitsch et al. 1996). Both techniques show an acceptably low CV for repeated measurements of skinfold thickness and/or whole-body resistance. The accuracy of these field methods in terms of measurements of FM depends on an appropriate prediction equation. Currently, numerous algorithms are in use in children to predict percentage FM (\% FM) from anthropometry and/or BIA measurements. For those authors who are involved in field studies it is unclear which prediction formulas are appropriate.

The Kiel Obesity Prevention Study (KOPS) is a crosssectional and longitudinal field study (Müller et al. 1998, 2001). The objective of this study is to reduce the prevalence and incidence of overweight and obesity in children and adolescents. Within KOPS, anthropometry and BIA are used to assess FM. Both techniques have been validated against so-called reference methods (e.g. densitometry, DXA) in small populations of children of different ages (Lohmann, 1986; Cordain et al. 1988; Houtkooper et al. 1989; Deurenberg et al. 1990; Kushner et al. 1992; Schaefer et al. 1994; Goran et al. 1996; Wabitsch et al. 1996). However, there is only limited experience with regard to their use in field studies. The present study compares BIA and anthropometric measurements of body fat in a group of 2286 prepubertal children from Kiel, north-west Germany.

\section{Methods}

\section{Subjects}

Within KOPS between 1996 and 1998 six different investigators assessed the nutritional status of 1146 boys and 1140 girls aged 5-7 years living in Kiel, north-west Germany. Anthropometric and BIA measurements were conducted weekly in the first month and every $14 \mathrm{~d}$ during the following weeks. The children were randomly sampled and examined in twenty-nine of a total of thirty-two primary schools in Kiel. The 2286 children represented $40 \%$ of the total population of children examined by the school physicians in the same time period. Age, gender, body weight and height, as well as socio-demographic data, suggested that this subpopulation was representative of the total population of 5-7-year-old children in Kiel (Mast et al. 1998). The procedures involved in the investigations were explained to all parents. All parents gave their informed written consent. The study protocol was approved by the local ethical committee of the Christian-AlbrechtsUniversity of Kiel.

\section{Anthropometry}

Anthropometric measurements were performed by six trained observers, according to standard procedures (Lohmann et al. 1988). Weight was measured to the nearest $0.1 \mathrm{~kg}$ using a calibrated balance-beam scale (Model 709; Seca Vogelt Halke, Hamburg, Germany) with subjects wearing light underwear only. Height was estimated to the nearest $5 \mathrm{~mm}$. Skinfold thickness was determined to the nearest $0.2 \mathrm{~mm}$ at the right triceps, biceps, subscapular and suprailiac sites, using a Lafayette skinfold caliper (Model 01127; Lafayette Instrument Company, Lafayette, IN 47903, USA) calibrated to exert a constant pressure of $10 \mathrm{~g} / \mathrm{mm}^{2}$. The between-observer CV for measurements of right triceps, biceps subscapular and suprailiac skinfolds were $5.7,8.0,13.2$ and $8.4 \%$ respectively ( $n$ 15). The corresponding within-observer $\mathrm{CV}$ for repeated (n 3) measurements in 150 children aged 5-7 years were $4 \cdot 2,6 \cdot 8,5 \cdot 1$ and $5 \cdot 3 \%$. FM was determined using age- and gender-specific formulas, according to Lohmann (1986) and Deurenberg et al. (1990), involving the log-transformed sum of right triceps, biceps, subscapular and suprailiac skinfolds for subjects aged $<12 \cdot 0$ years (Table 1). In order to compare values derived from different methods, we used \% FM from anthropometry according to Lohmann (1986) as an example.

In a subgroup of twelve children (eight boys, four girls) aged 6.3 years with BMI of 15.1 and $15.9 \mathrm{~kg} / \mathrm{m}^{2}$ for boys and girls respectively we examined the relationship between measurements of body proportions (i.e. lengths of arms and legs, elbow breadth and arm, waist and hip circumference) and resistance values.

Table 1. Algorithms used for estimating fat mass (FM) from anthropometric and bioelectrical impedance analysis data, as proposed by different authors

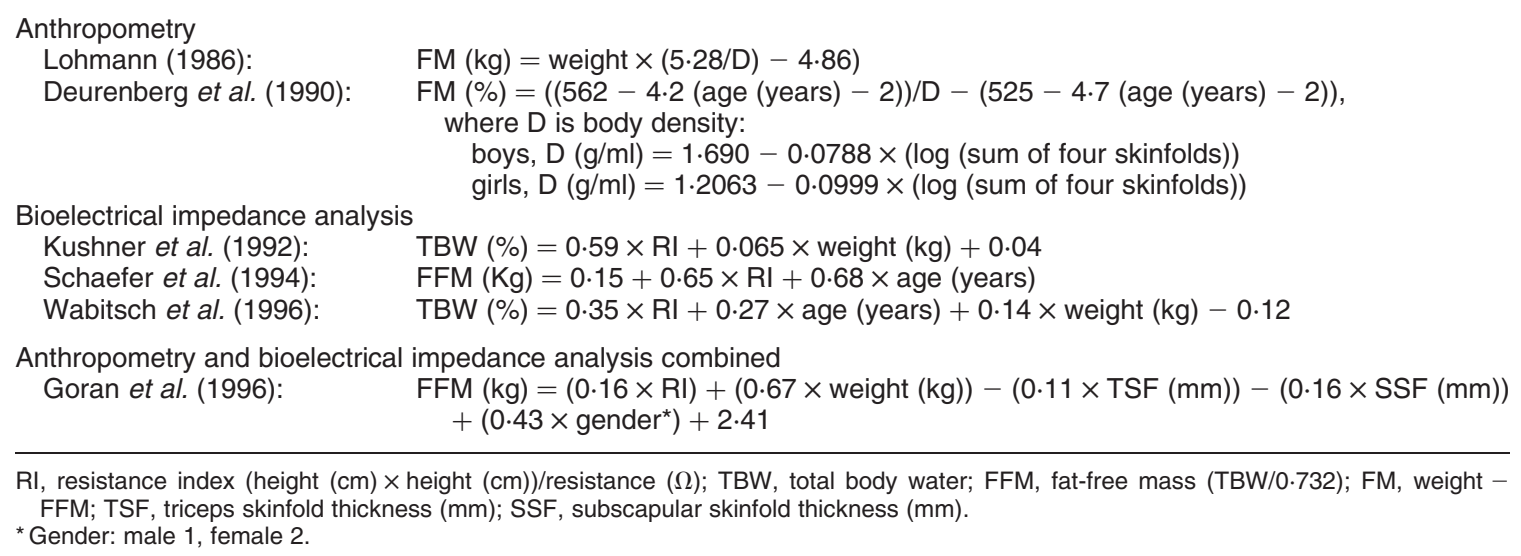




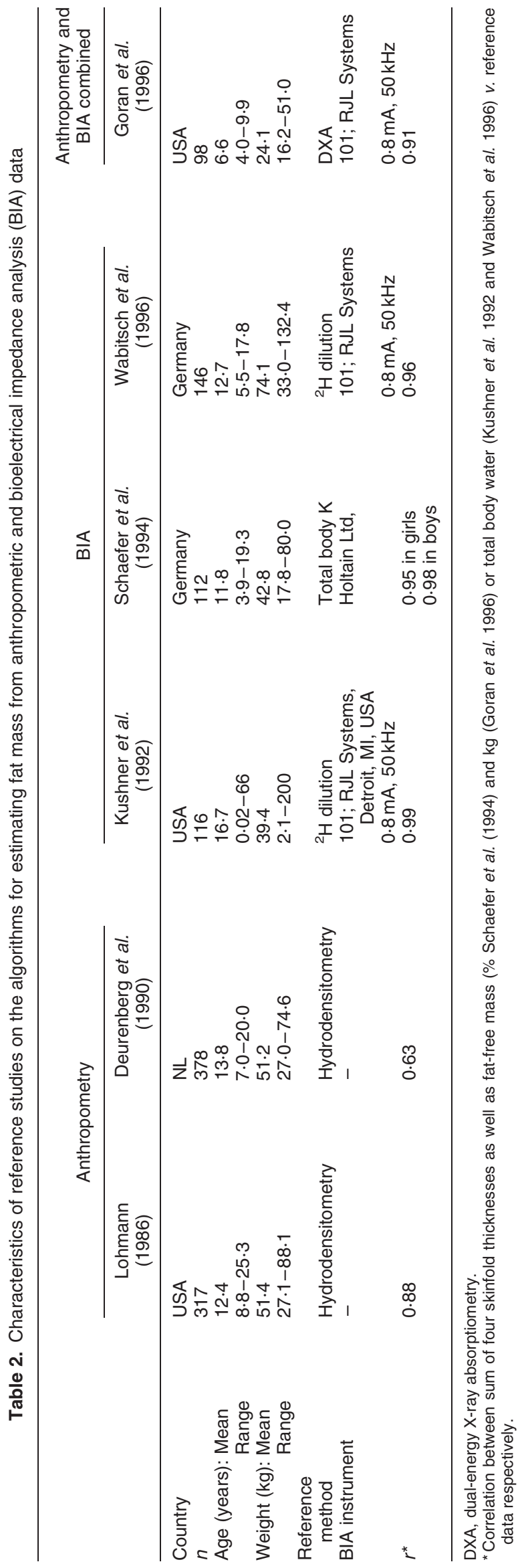

\section{Bioelectrical impedance analysis}

Measurements of whole-body impedance in children have been described previously (Mast et al. 1998). Measurements were performed at a single frequency $(50 \mathrm{kHz})$ with one pair of electrodes appropriately placed on the dorsal surfaces of the right hand and a second pair of electrodes placed on the right foot using Multi-Frequency-Analyzers (2000-M; Data Input GmbH, Frankfurt am Main, Germany; Müller, 1998). Three different analysers were cross-calibrated in ten children aged 5-7 years, including disconnected electrodes, and the difference in the measurements obtained using the different analysers was found to be $<1 \%$. With the subjects lying in a supine position, measurements were performed while the hands and feet were extended at about $30^{\circ}$ from the side of the trunk. The CV for repeated (n 3) estimates of resistance $(\Omega)$ and reactance $(\Omega)$ in ten children aged 5-7 years were 1.0 and $2.1 \%$ respectively, resulting in a CV of 1.5 in $\% \mathrm{FM}$.

For data analysis different algorithms have been proposed for children. The prediction equations are based on resistance, height, weight, age and gender (Table 1 shows examples of algorithms). In addition, one algorithm also includes anthropometric measures in the calculation of fat-free mass (FFM) from BIA data (Goran et al. 1996). FM was calculated from the difference between body weight and FFM. Differences between the algorithms result from variations in age and weight in the reference population, as well as the use of different reference methods (Table 2). We assumed that the reference population studied by Schaefer et al. (1994) is very close to our study population (age and weight of reference population).

\section{Statistical analyses}

All analyses were performed using Excel 5.0 or SPSS for windows (SPSS Inc., Chicago, IL, USA). The data are presented as medians and interquartile ranges or as means and ranges of minimum to maximum. Basic statistics included $U$ test for comparison between gender groups. Multiple regression analysis were used to assess the relationship between measurements of body proportions and resistance values. Simple linear correlation analyses were used to assess the relationship between the different methods for estimating FM. In addition to the standard procedure, a Bland \& Altman (1986) plot was used to compare the differences between the two methods. A positive difference indicated an overestimation of \% FM, whereas a negative difference suggested an underestimation of $\%$ FM.

\section{Results}

Table 3 shows anthropometric characteristics as well as BIA data for the KOPS population. There were significant gender differences in height, weight, skinfold thickness and impedance as well as the estimates of \% FM. We found no gender differences in BMI. Using anthropometry, skinfold thicknesses were greater for girls than for boys (Table 3 ). For anthropometric measurements there were age-depen- 
Table 3. Anthropometric and bioelectrical impedance analysis measurements in 2286 5-7-year-old boys and girls in Kiel, north-west Germany $\dagger$ (Medians and interquartile ranges, with no. of subjects in parentheses)

\begin{tabular}{|c|c|c|c|c|c|c|c|c|c|c|c|c|}
\hline & \multicolumn{4}{|c|}{ Children aged 5 years } & \multicolumn{4}{|c|}{ Children aged 6 years } & \multicolumn{4}{|c|}{ Children aged 7 years } \\
\hline & \multicolumn{2}{|c|}{ Boys (23) } & \multicolumn{2}{|c|}{ Girls (15) } & \multicolumn{2}{|c|}{ Boys (765) } & \multicolumn{2}{|c|}{ Girls (819) } & \multicolumn{2}{|c|}{ Boys (357) } & \multicolumn{2}{|c|}{ Girls (306) } \\
\hline & Median & $\begin{array}{l}\text { Interquartiles } \\
\text { range }\end{array}$ & Median & $\begin{array}{l}\text { Interquartiles } \\
\text { range }\end{array}$ & Median & $\begin{array}{l}\text { Interquartiles } \\
\text { range }\end{array}$ & Median & $\begin{array}{l}\text { Interquartiles } \\
\text { range }\end{array}$ & Median & $\begin{array}{l}\text { Interquartiles } \\
\text { range }\end{array}$ & Median & $\begin{array}{l}\text { Interquartiles } \\
\text { range }\end{array}$ \\
\hline $\begin{array}{l}\text { Age (years) } \\
\text { Anthronometry }\end{array}$ & $5 \cdot 02$ & 0.2 & $5 \cdot 5$ & 0.2 & $6 \cdot 1$ & 0.4 & $6 \cdot 1$ & 0.4 & $6 \cdot 7$ & 0.2 & $6 \cdot 6$ & 0.2 \\
\hline \multicolumn{13}{|l|}{$\begin{array}{l}\text { Anthropometry } \\
\text { Measurements }\end{array}$} \\
\hline Height $(m)$ & $1 \cdot 20$ & 0.1 & $1 \cdot 17^{\star \star \star}$ & 0.1 & $1 \cdot 20$ & 0.1 & $1 \cdot 19^{\star \star \star}$ & 0.1 & 1.23 & 0.1 & $1 \cdot 22^{\star * \star}$ & 0.1 \\
\hline Weight (kg) & 21.6 & 3.2 & $19 \cdot 5^{\star \star}$ & 8.2 & $22 \cdot 3$ & 4.5 & $22 \cdot 0^{\star \star}$ & 4.5 & 23.5 & 4.9 & $23 \cdot 0^{\star \star}$ & 4.6 \\
\hline $\mathrm{BMI}\left(\mathrm{kg} / \mathrm{m}^{2}\right)$ & $14 \cdot 8$ & 1.4 & 14.5 & 2.9 & $15 \cdot 5$ & $2 \cdot 0$ & $15 \cdot 6$ & $2 \cdot 1$ & $15 \cdot 6$ & $2 \cdot 0$ & $15 \cdot 5$ & 1.9 \\
\hline Weight:hip & 0.92 & 0.1 & $0.90^{\star \star \star}$ & 0.1 & 0.91 & 0.1 & $0.89^{\star \star \star}$ & 0.1 & 0.90 & 0.1 & $0.88^{\star \star \star}$ & 0.1 \\
\hline TSF $(\mathrm{mm})$ & 9.0 & 3.2 & $12 \cdot 0^{\star \star \star}$ & 5.4 & $10 \cdot 3$ & $3 \cdot 7$ & $11 \cdot 3^{\star * \star}$ & 4.6 & 9.8 & 4.3 & $11 \cdot 6^{\star \star \star}$ & 4.4 \\
\hline $\mathrm{BSF}(\mathrm{mm})$ & $5 \cdot 0$ & 1.8 & $7 \cdot 3^{\star \star \star}$ & 5.5 & $5 \cdot 6$ & 3.4 & $6 \cdot 3^{\star \star \star}$ & 3.3 & $5 \cdot 3$ & $3 \cdot 3$ & $6 \cdot 0^{\star \star \star \star}$ & 3.6 \\
\hline SIF $(\mathrm{mm})$ & $5 \cdot 3$ & $2 \cdot 8$ & $7 \cdot 6^{\star \star \star}$ & 4.4 & $6 \cdot 3$ & $4 \cdot 3$ & $8 \cdot 0^{\star \star \star}$ & $5 \cdot 4$ & $6 \cdot 0$ & 4.4 & $7 \cdot 6^{\star \star \star}$ & 4.7 \\
\hline $\operatorname{SSF}(\mathrm{mm})$ & $5 \cdot 0$ & $2 \cdot 0$ & $6 \cdot 6^{\star \star \star}$ & $3 \cdot 2$ & $5 \cdot 6$ & $2 \cdot 4$ & $6 \cdot 3^{\star \star \star}$ & 3.2 & $5 \cdot 0$ & $2 \cdot 3$ & $6 \cdot 0^{\star \star \star}$ & 3.0 \\
\hline $\begin{array}{l}\text { Sum of four } \\
\text { skinfolds }(\mathrm{mm})\end{array}$ & $24 \cdot 0$ & $8 \cdot 8$ & $33 \cdot 3^{\star \star \star}$ & $20 \cdot 7$ & $28 \cdot 2$ & $12 \cdot 1$ & $31 \cdot 7^{\star \star \star}$ & $14 \cdot 1$ & $26 \cdot 8$ & $10 \cdot 8$ & $30 \cdot 5^{\star \star \star}$ & $14 \cdot 1$ \\
\hline \multicolumn{13}{|l|}{ Fat mass: $\%$} \\
\hline $\mathrm{FM}_{\mathrm{J}} \mathrm{Obmann}$ & $12 \cdot 0$ & 5.9 & $14 \cdot 9^{*}$ & 11.9 & $14 \cdot 6$ & 6.9 & $13 \cdot 8^{*}$ & 8.9 & $13 \cdot 8$ & 6.5 & $13 \cdot 1^{*}$ & 8.9 \\
\hline $\begin{array}{c}\mathrm{FM}_{\text {Deurenberg }} \\
\mathrm{Kg}\end{array}$ & 8.5 & $4 \cdot 6$ & $11 \cdot 1$ & $13 \cdot 4$ & $10 \cdot 8$ & $7 \cdot 1$ & $10 \cdot 3$ & $9 \cdot 0$ & 10.5 & 6.5 & $10 \cdot 1$ & $9 \cdot 1$ \\
\hline $\mathrm{FM}_{\text {Lohmann }}$ & $2 \cdot 6$ & 1.8 & $2 \cdot 5^{\star}$ & 3.0 & $3 \cdot 2$ & $2 \cdot 0$ & $3 \cdot 0$ & $2 \cdot 5^{*}$ & $3 \cdot 1$ & $2 \cdot 0$ & $20 \cdot 1^{*}$ & $3 \cdot 1$ \\
\hline $\mathrm{FM}_{\text {Deurenberg }}$ & $1 \cdot 7$ & 1.4 & 1.9 & $3 \cdot 0$ & $2 \cdot 4$ & 1.9 & $2 \cdot 2$ & $2 \cdot 3$ & $2 \cdot 4$ & 1.9 & $2 \cdot 2$ & $2 \cdot 4$ \\
\hline \multicolumn{13}{|l|}{ Fat-free mass: \% } \\
\hline FFM Lohmann & $88 \cdot 0$ & 5.9 & $85 \cdot 2$ & $11 \cdot 9$ & $85 \cdot 4$ & 6.9 & $86 \cdot 2$ & 8.9 & $86 \cdot 2$ & 6.5 & $87 \cdot 0$ & 8.9 \\
\hline $\begin{array}{c}\text { FFM }_{\text {Deurenberg }} \\
\mathrm{Kg}\end{array}$ & 91.5 & $4 \cdot 6$ & 89.0 & $13 \cdot 4$ & $89 \cdot 2$ & $7 \cdot 1$ & $89 \cdot 7$ & $9 \cdot 0$ & 89.5 & 6.5 & 89.9 & $9 \cdot 1$ \\
\hline FFM Lohmann & $19 \cdot 0$ & 3.5 & $17 \cdot 3$ & 6.5 & $19 \cdot 1$ & $3 \cdot 3$ & 18.9 & 3.0 & $20 \cdot 3$ & 3.6 & 20.1 & $3 \cdot 1$ \\
\hline FFM & $20 \cdot 0$ & 3.7 & $17 \cdot 7^{\star \star \star}$ & $7 \cdot 8$ & $20 \cdot 0$ & 3.4 & $19 \cdot 7^{\star \star \star}$ & $3 \cdot 1$ & $21 \cdot 0$ & 3.8 & $20 \cdot 9^{\star \star \star}$ & 3.2 \\
\hline
\end{tabular}


BIA

Measurements

\begin{tabular}{|c|c|c|c|c|c|c|c|c|c|c|c|c|}
\hline $\mathrm{R}(\Omega)$ & 768 & $80 \cdot 5$ & $805^{\star \star \star}$ & $87 \cdot 3$ & 749 & 101.9 & $788 \cdot 4^{\star \star \star}$ & $95 \cdot 3$ & 739 & $94 \cdot 1$ & 791 & $118 \cdot 3$ \\
\hline $\mathrm{Xc}(\Omega)$ & 68 & $6 \cdot 0$ & $73^{\star \star \star}$ & $9 \cdot 1$ & 68.0 & $10 \cdot 0$ & $72 \cdot 0^{\star * *}$ & 12.9 & $67 \cdot 1$ & $11 \cdot 2$ & 72 & $12 \cdot 0$ \\
\hline Phase angle $\alpha$ & $5 \cdot 0$ & $0 \cdot 6$ & $5 \cdot 2$ & 0.6 & $5 \cdot 1$ & 0.6 & $5 \cdot 2$ & 0.7 & $5 \cdot 2$ & 0.7 & $5 \cdot 2$ & 0.7 \\
\hline $\begin{array}{l}\mathrm{RI}\left(\mathrm{cm}^{2} / \Omega\right) \\
\text { t mass: \% }\end{array}$ & $18 \cdot 8$ & $2 \cdot 9$ & $17 \cdot 1^{\star \star \star}$ & $3 \cdot 7$ & $19 \cdot 2$ & $3 \cdot 8$ & $18 \cdot 0^{\star \star *}$ & $2 \cdot 2$ & $20 \cdot 4$ & 3.7 & $19 \cdot 0^{\star \star \star}$ & 4 \\
\hline $\mathrm{FM}_{\text {Schaefer }}$ & $27 \cdot 1$ & $6 \cdot 4$ & $29 \cdot 2^{\star \star \star}$ & $15 \cdot 4$ & $25 \cdot 4$ & $8 \cdot 2$ & $27 \cdot 6^{* * *}$ & $9 \cdot 8$ & $23 \cdot 7$ & $8 \cdot 8$ & $27 \cdot 0^{\star \star \star}$ & \\
\hline FM Kushner & $20 \cdot 3$ & 6.9 & $25 \cdot 2^{\star \star \star}$ & $15 \cdot 8$ & $22 \cdot 8$ & $8 \cdot 3$ & $25 \cdot 9^{\star \star *}$ & 9.5 & $22 \cdot 0$ & $9 \cdot 0$ & $26 \cdot 0^{* * *}$ & \\
\hline $\mathrm{FM}_{\text {Wabitsch }}$ & 31.4 & 4.6 & $33 \cdot 4^{\star \star *}$ & $10 \cdot 2$ & $30 \cdot 8$ & 5.4 & $32 \cdot 5^{\star \star *}$ & $6 \cdot 4$ & 29.9 & $6 \cdot 2$ & $32 \cdot 2^{* \star *}$ & 5. \\
\hline $\mathrm{FM}_{\mathrm{Goran}}$ & $13 \cdot 8$ & $4 \cdot 0$ & $18 \cdot 6^{\star \star \star}$ & $6 \cdot 3$ & $15 \cdot 8$ & 4.7 & $19 \cdot 3^{\star \star \star}$ & $5 \cdot 5$ & $15 \cdot 1$ & $4 \cdot 6$ & $19 \cdot 1^{* \star *}$ & \\
\hline $\mathrm{FM}_{\text {Schaefer }}$ & $5 \cdot 9$ & $1 \cdot 4$ & $5 \cdot 0^{\star \star \star}$ & 4.5 & $5 \cdot 5$ & $2 \cdot 8$ & $6 \cdot 0^{\star \star \star}$ & $3 \cdot 3$ & $5 \cdot 4$ & $2 \cdot 9$ & $6 \cdot 1^{\star *}$ & \\
\hline $\mathrm{FM}_{\text {Kushner }}$ & $4 \cdot 6$ & $1 \cdot 8$ & $5 \cdot 2^{\star \star *}$ & $4 \cdot 0$ & 4.9 & $2 \cdot 4$ & $5 \cdot 6^{\star \star \star}$ & $2 \cdot 7$ & $5 \cdot 0$ & $2 \cdot 6$ & $5 \cdot 9^{\star \star *}$ & \\
\hline FM $_{\text {Wabitsch }}$ & 6.9 & $1 \cdot 1$ & $5 \cdot 8^{*}$ & $3 \cdot 7$ & $6 \cdot 8$ & $2 \cdot 3$ & $7 \cdot 1^{*}$ & $2 \cdot 6$ & 6.9 & $2 \cdot 3$ & $7 \cdot 3^{*}$ & \\
\hline $\mathrm{FM}_{\text {Goran }}$ & 2.9 & $1 \cdot 1$ & $3 \cdot 8^{\star \star \star}$ & $2 \cdot 5$ & 3.5 & 1.6 & $4 \cdot 2^{\star \star \star}$ & 1.9 & 3.5 & 1.5 & $4 \cdot 4^{\star \star \star}$ & \\
\hline \multicolumn{13}{|l|}{ t-free mass: \% } \\
\hline FFMSchaefer & $72 \cdot 9$ & $6 \cdot 4$ & $70 \cdot 8^{\star \star *}$ & 15.4 & 74.6 & $8 \cdot 2$ & $72 \cdot 4$ & $9 \cdot 8$ & $76 \cdot 3$ & $8 \cdot 8$ & $73 \cdot 0^{* \star *}$ & \\
\hline FFM Kushner & $79 \cdot 7$ & 6.9 & $74 \cdot 8^{\star \star \star}$ & $15 \cdot 8$ & $77 \cdot 2$ & $8 \cdot 3$ & $74 \cdot 1$ & 9.5 & $78 \cdot 0$ & $9 \cdot 0$ & $74 \cdot 0^{\star \star \star}$ & \\
\hline FFM Wabitsch & 68.6 & $4 \cdot 6$ & $66 \cdot 6^{\star \star *}$ & $10 \cdot 2$ & $69 \cdot 2$ & 5.4 & 67.5 & 6.4 & $70 \cdot 1$ & $6 \cdot 2$ & $67 \cdot 9^{* * *}$ & \\
\hline FFM $_{\text {Goran }} \mathrm{Kg}$ & $86 \cdot 3$ & $4 \cdot 0$ & $81 \cdot 4^{\star * *}$ & $6 \cdot 3$ & 84.2 & 4.7 & $80 \cdot 7$ & $5 \cdot 5$ & 84.9 & $4 \cdot 6$ & $80 \cdot 9^{\star \star \star}$ & \\
\hline $\mathrm{FFM}_{\text {Schaefer }}$ & $15 \cdot 7$ & $1 \cdot 7$ & $14 \cdot 9^{\star \star *}$ & $2 \cdot 4$ & $16 \cdot 7$ & $2 \cdot 6$ & $16 \cdot 0$ & $2 \cdot 1$ & $18 \cdot 0$ & 2.5 & $17 \cdot 0^{\star * *}$ & \\
\hline FFM Kushner & $17 \cdot 1$ & $2 \cdot 6$ & $15 \cdot 3^{\star \star \star}$ & 3.7 & 17.5 & 3.4 & $16 \cdot 5$ & 2.9 & $18 \cdot 6$ & 3.5 & $17 \cdot 4^{\star \star \star}$ & \\
\hline FFM Wabitsch & $14 \cdot 7$ & $2 \cdot 0$ & $13 \cdot 3^{*}$ & $3 \cdot 3$ & $15 \cdot 6$ & $2 \cdot 3$ & $15 \cdot 0$ & 2.4 & $16 \cdot 6$ & $2 \cdot 7$ & $15 \cdot 9^{\star}$ & \\
\hline FFM & 18.4 & 2.9 & $16 \cdot 1^{\star \star *}$ & $5 \cdot 9$ & $18 \cdot 8$ & $3 \cdot 1$ & $17 \cdot 7$ & 3.0 & $20 \cdot 1$ & 3.5 & $18 \cdot 9^{* * *}$ & \\
\hline
\end{tabular}

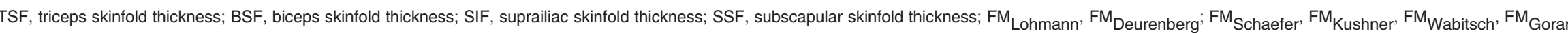
fat mass estimated based on algorithms proposed by Lohmann (1986), Deurenberg et al. (1990), Schaefer et al. (1994), Kushner et al. (1992), Wabitsch et al. (1996) and Goran et al. (1996) respectively (for details, see Tables 1 and 2); FFM Lohmann, $_{\text {FFM }}$ Deurenberg, FFM $_{\text {Schaefer, }}$ FFM $_{\text {Kushner, }}$ FFM Wabitsch, FFM $_{\text {Goran }}$, fat-free mass estimated based on algorithms proposed by Lohmann (1986), Deurenberg et al. (1990), Schaefer et al. (1994), Kushner et al. (1992), Wabitsch et al. (1996) and Goran et al. (1996) respectively (for details, see Tables 1 and 2); R, resistance from BIA; Xc, reactance from BIA; RI, resistance index (height $(\mathrm{cm}) \times$ height $(\mathrm{cm})) / \mathrm{R}(\Omega)$

Median values were significantly different from those for boys: * $P \leq 0.05,{ }^{* * *} P \leq 0.01, * \star * x \leq 0.001$

†For details of subjects and procedures, see p. 164. 
Table 4. 10th, 50th, and 90th percentiles of anthropometric and bioelectrical impedance analysis(BIA) measurements as well as fat mass derived using both techniques for $6-7-y e a r-o l d ~$ boys and girls from Kiel, north-west Germany

(Percentiles were not calculated for 5-year-old children because of the limited no. of children studied in this age-group)

\begin{tabular}{|c|c|c|c|c|c|c|c|c|c|c|c|c|}
\hline \multirow{3}{*}{$\begin{array}{l}\text { Age group... } \\
n \ldots \\
\text { Percentile... }\end{array}$} & \multicolumn{6}{|c|}{6 years old } & \multicolumn{6}{|c|}{7 years old } \\
\hline & \multicolumn{3}{|c|}{$\begin{array}{l}\text { Boys } \\
765\end{array}$} & \multicolumn{3}{|c|}{$\begin{array}{l}\text { Girls } \\
819\end{array}$} & \multicolumn{3}{|c|}{$\begin{array}{l}\text { Boys } \\
354\end{array}$} & \multicolumn{3}{|c|}{$\begin{array}{l}\text { Girls } \\
303\end{array}$} \\
\hline & 10th & 50th & 90th & 10th & 50th & 90th & 10th & 50th & 90th & 10th & 50th & 90th \\
\hline \multicolumn{13}{|l|}{ Anthropometric measurements } \\
\hline Height $(\mathrm{m})$ & $1 \cdot 13$ & $1 \cdot 2$ & $1 \cdot 26$ & $1 \cdot 13$ & 1.19 & $1 \cdot 26$ & $1 \cdot 16$ & $1 \cdot 23$ & $1 \cdot 30$ & $1 \cdot 16$ & $1 \cdot 22$ & 1.29 \\
\hline Weight (kg) & $19 \cdot 0$ & $22 \cdot 3$ & $28 \cdot 7$ & $18 \cdot 5$ & $22 \cdot 0$ & $27 \cdot 7$ & $20 \cdot 0$ & 23.5 & 30.5 & $19 \cdot 5$ & 23.0 & $29 \cdot 0$ \\
\hline $\operatorname{BMI}\left(\mathrm{kg} / \mathrm{m}^{2}\right)$ & $14 \cdot 1$ & $15 \cdot 5$ & 18.5 & 13.8 & $15 \cdot 6$ & 18.4 & 14.0 & $15 \cdot 6$ & $18 \cdot 7$ & 14.0 & $15 \cdot 5$ & $18 \cdot 4$ \\
\hline Weight:hip & 0.83 & 0.91 & 0.97 & 0.82 & 0.89 & 0.96 & 0.83 & 0.90 & 0.96 & 0.80 & 0.88 & 0.95 \\
\hline TSF $(\mathrm{mm})$ & $7 \cdot 3$ & $10 \cdot 3$ & $15 \cdot 3$ & 8.0 & 11.3 & $16 \cdot 3$ & $6 \cdot 8$ & 9.8 & $15 \cdot 0$ & 8.0 & 11.6 & $15 \cdot 6$ \\
\hline $\mathrm{BSF}(\mathrm{mm})$ & $3 \cdot 3$ & $5 \cdot 6$ & $10 \cdot 3$ & 4.0 & $6 \cdot 3$ & $11 \cdot 0$ & 3.0 & $5 \cdot 3$ & $10 \cdot 2$ & $3 \cdot 6$ & $6 \cdot 0$ & $10 \cdot 6$ \\
\hline $\operatorname{SIF}(\mathrm{mm})$ & $3 \cdot 8$ & $6 \cdot 3$ & $14 \cdot 0$ & $4 \cdot 3$ & $8 \cdot 0$ & $15 \cdot 3$ & $3 \cdot 6$ & $6 \cdot 0$ & $14 \cdot 2$ & $4 \cdot 3$ & $7 \cdot 6$ & $15 \cdot 0$ \\
\hline $\mathrm{SSF}(\mathrm{mm})$ & 4.0 & $5 \cdot 6$ & 9.9 & 4.0 & $6 \cdot 3$ & $10 \cdot 7$ & 3.4 & $5 \cdot 0$ & $9 \cdot 0$ & 4.0 & $6 \cdot 0$ & $10 \cdot 7$ \\
\hline Sum of four skinfolds (mm) & $20 \cdot 0$ & $28 \cdot 2$ & $48 \cdot 6$ & $22 \cdot 3$ & $31 \cdot 7$ & $51 \cdot 1$ & $18 \cdot 6$ & $26 \cdot 8$ & $46 \cdot 6$ & $22 \cdot 6$ & $30 \cdot 5$ & $51 \cdot 3$ \\
\hline \multicolumn{13}{|l|}{ Fat mass: \% } \\
\hline FM Lohmann & $9 \cdot 1$ & $14 \cdot 6$ & $23 \cdot 6$ & $6 \cdot 8$ & $13 \cdot 8$ & $23 \cdot 8$ & $8 \cdot 0$ & $13 \cdot 8$ & $22 \cdot 9$ & $7 \cdot 0$ & $13 \cdot 1$ & 23.9 \\
\hline $\begin{array}{c}\mathrm{FM}_{\text {Deurenberg }} \\
\mathrm{kg}\end{array}$ & $5 \cdot 2$ & $10 \cdot 8$ & $20 \cdot 2$ & 3.7 & $10 \cdot 3$ & $20 \cdot 4$ & 4.7 & $10 \cdot 5$ & $19 \cdot 8$ & 3.9 & $10 \cdot 1$ & $20 \cdot 9$ \\
\hline $\mathrm{FM}_{\text {Lohmann }}$ & 1.9 & $3 \cdot 2$ & $6 \cdot 3$ & 1.3 & 3.0 & $6 \cdot 3$ & 1.7 & $3 \cdot 1$ & 6.9 & 1.4 & $2 \cdot 9$ & $7 \cdot 0$ \\
\hline FM Deurenberg & $1 \cdot 1$ & $2 \cdot 4$ & $5 \cdot 4$ & 0.8 & $2 \cdot 2$ & $5 \cdot 3$ & 1.0 & $2 \cdot 4$ & 5.9 & 0.8 & $2 \cdot 2$ & $6 \cdot 2$ \\
\hline \multicolumn{13}{|l|}{ Fat-free mass: \% } \\
\hline FFM Lohmann & $76 \cdot 5$ & 85.4 & $90 \cdot 9$ & $76 \cdot 2$ & $86 \cdot 2$ & 93.3 & $77 \cdot 1$ & $86 \cdot 2$ & $92 \cdot 1$ & $76 \cdot 1$ & $87 \cdot 0$ & 93.0 \\
\hline $\begin{array}{r}\mathrm{FFM}_{\text {Deurenberg }} \\
\mathrm{kg}\end{array}$ & $79 \cdot 8$ & $89 \cdot 2$ & $94 \cdot 8$ & $79 \cdot 6$ & $89 \cdot 7$ & $96 \cdot 3$ & $80 \cdot 2$ & $89 \cdot 5$ & $95 \cdot 3$ & $79 \cdot 2$ & $89 \cdot 9$ & $96 \cdot 1$ \\
\hline FFM ${ }_{\text {Lohmann }}$ & $16 \cdot 3$ & $19 \cdot 1$ & $22 \cdot 8$ & $16 \cdot 3$ & $18 \cdot 9$ & $22 \cdot 1$ & $17 \cdot 6$ & $20 \cdot 3$ & 23.9 & $17 \cdot 3$ & $20 \cdot 1$ & 23.5 \\
\hline FFM $_{\text {Deurenberg }}$ & $17 \cdot 1$ & $20 \cdot 0$ & $23 \cdot 7$ & $17 \cdot 0$ & $19 \cdot 7$ & $23 \cdot 1$ & $18 \cdot 2$ & $21 \cdot 0$ & $24 \cdot 8$ & $18 \cdot 2$ & $20 \cdot 9$ & $24 \cdot 4$ \\
\hline
\end{tabular}




\begin{tabular}{|c|c|c|c|c|c|c|c|c|c|c|c|c|}
\hline $\mathrm{R}(\Omega)$ & $660 \cdot 3$ & 749 & 850 & 702.9 & 788.4 & $900 \cdot 8$ & 653.6 & 739 & $831 \cdot 1$ & 695.4 & $791 \cdot 0$ & $888 \cdot 8$ \\
\hline $\mathrm{Xc}(\Omega)$ & 58 & 68 & $79 \cdot 7$ & 61 & 72 & 85 & 58 & $67 \cdot 1$ & 78.7 & $61 \cdot 8$ & 72 & 84.9 \\
\hline Phase angle $\alpha$ & $4 \cdot 6$ & $5 \cdot 1$ & 5.9 & 4.5 & $5 \cdot 2$ & $5 \cdot 9$ & 4.5 & $5 \cdot 2$ & $6 \cdot 0$ & 4.6 & $5 \cdot 2$ & $6 \cdot 1$ \\
\hline $\mathrm{RI}\left(\mathrm{cm}^{2} / \Omega\right)$ & $15 \cdot 6$ & $19 \cdot 2$ & 23.2 & 14.9 & $18 \cdot 0$ & 21.3 & $16 \cdot 9$ & $20 \cdot 4$ & $24 \cdot 8$ & $15 \cdot 7$ & $19 \cdot 0$ & $22 \cdot 8$ \\
\hline \multicolumn{13}{|l|}{ Fat mass: \% } \\
\hline $\mathrm{FM}_{\text {Schaefer }}$ & $17 \cdot 5$ & $25 \cdot 3$ & $35 \cdot 6$ & $19 \cdot 3$ & $27 \cdot 6$ & 37.9 & $16 \cdot 5$ & $23 \cdot 7$ & 34.5 & $19 \cdot 4$ & $27 \cdot 0$ & $37 \cdot 0$ \\
\hline FM Kushner & $14 \cdot 3$ & $22 \cdot 8$ & $31 \cdot 7$ & $17 \cdot 4$ & $25 \cdot 9$ & 34.6 & $13 \cdot 7$ & $22 \cdot 0$ & $30 \cdot 4$ & $17 \cdot 7$ & $26 \cdot 0$ & 33.9 \\
\hline FM Wabitsch & $25 \cdot 5$ & $30 \cdot 8$ & $37 \cdot 6$ & $26 \cdot 8$ & 32.5 & $39 \cdot 2$ & $24 \cdot 7$ & 29.9 & $36 \cdot 6$ & $26 \cdot 8$ & $32 \cdot 2$ & $38 \cdot 8$ \\
\hline${ }_{\mathrm{Fg}}^{\mathrm{F} \mathrm{Goran}}$ & 11.9 & $15 \cdot 8$ & $22 \cdot 2$ & $15 \cdot 2$ & $19 \cdot 3$ & $25 \cdot 6$ & $11 \cdot 5$ & $15 \cdot 1$ & $21 \cdot 5$ & $15 \cdot 0$ & $19 \cdot 1$ & 24.9 \\
\hline $\mathrm{FM}_{\text {Schaefer }}$ & $3 \cdot 4$ & $5 \cdot 5$ & $10 \cdot 0$ & 3.7 & $6 \cdot 0$ & $10 \cdot 4$ & 3.4 & 5.4 & $10 \cdot 1$ & $3 \cdot 9$ & $6 \cdot 1$ & $11 \cdot 0$ \\
\hline FM Kushner & 3.0 & 4.9 & $8 \cdot 4$ & 3.5 & $5 \cdot 6$ & $9 \cdot 1$ & $3 \cdot 1$ & $5 \cdot 0$ & $8 \cdot 6$ & $3 \cdot 8$ & 5.9 & 9.5 \\
\hline $\mathrm{FM}_{\text {Wabitsch }}$ & $5 \cdot 1$ & $6 \cdot 8$ & $10 \cdot 5$ & $5 \cdot 2$ & $7 \cdot 1$ & $10 \cdot 8$ & $5 \cdot 2$ & $6 \cdot 9$ & $11 \cdot 0$ & $5 \cdot 4$ & $7 \cdot 3$ & 11.4 \\
\hline $\mathrm{FM}_{\text {Goran }}$ & $2 \cdot 4$ & 3.5 & $6 \cdot 2$ & $2 \cdot 9$ & $4 \cdot 2$ & $6 \cdot 8$ & $2 \cdot 4$ & $3 \cdot 6$ & $6 \cdot 4$ & $3 \cdot 2$ & $4 \cdot 4$ & $7 \cdot 0$ \\
\hline \multicolumn{13}{|l|}{ Fat-free mass: \% } \\
\hline $\mathrm{FM}_{\text {Schaefer }}$ & $64 \cdot 3$ & $74 \cdot 6$ & 82.4 & $62 \cdot 1$ & $72 \cdot 4$ & $80 \cdot 7$ & $65 \cdot 5$ & $76 \cdot 3$ & 83.5 & 63.0 & $73 \cdot 0$ & $80 \cdot 6$ \\
\hline $\mathrm{FM}_{\text {Kushner }}$ & $68 \cdot 3$ & $77 \cdot 2$ & $85 \cdot 6$ & 65.4 & $74 \cdot 1$ & $82 \cdot 7$ & $69 \cdot 6$ & $78 \cdot 0$ & $86 \cdot 3$ & $66 \cdot 2$ & $74 \cdot 0$ & $82 \cdot 3$ \\
\hline FM Mabitsch $_{\text {Wabs }}$ & $62 \cdot 4$ & $69 \cdot 2$ & 74.4 & $60 \cdot 8$ & $67 \cdot 5$ & $73 \cdot 2$ & 63.4 & $70 \cdot 1$ & $75 \cdot 3$ & $61 \cdot 2$ & 67.9 & $73 \cdot 2$ \\
\hline $\mathrm{FM}_{\mathrm{Goran}}$ & $77 \cdot 8$ & $84 \cdot 2$ & $88 \cdot 1$ & $74 \cdot 4$ & $80 \cdot 7$ & $84 \cdot 8$ & $78 \cdot 6$ & 84.9 & 88.5 & $75 \cdot 2$ & $80 \cdot 9$ & $85 \cdot 0$ \\
\hline \multicolumn{13}{|l|}{ kg } \\
\hline FFMSchaefer & $14 \cdot 5$ & $16 \cdot 7$ & $19 \cdot 4$ & $14 \cdot 0$ & $16 \cdot 0$ & $18 \cdot 2$ & $15 \cdot 7$ & $18 \cdot 0$ & $20 \cdot 8$ & $14 \cdot 9$ & $17 \cdot 0$ & $19 \cdot 6$ \\
\hline FFM Kushner & $14 \cdot 4$ & $17 \cdot 5$ & $21 \cdot 3$ & $13 \cdot 8$ & $16 \cdot 5$ & $19 \cdot 5$ & $15 \cdot 5$ & $18 \cdot 6$ & $22 \cdot 6$ & $14 \cdot 4$ & $17 \cdot 4$ & $20 \cdot 9$ \\
\hline FFM $_{\text {Wabitsch }}$ & $13 \cdot 3$ & $15 \cdot 6$ & $18 \cdot 6$ & $12 \cdot 8$ & $15 \cdot 0$ & $17 \cdot 4$ & $14 \cdot 3$ & $16 \cdot 7$ & $19 \cdot 7$ & $13 \cdot 5$ & $15 \cdot 9$ & $18 \cdot 6$ \\
\hline FFM $_{\text {Goran }}$ & $16 \cdot 2$ & $18 \cdot 8$ & $22 \cdot 8$ & $15 \cdot 2$ & $17 \cdot 7$ & $21 \cdot 2$ & $17 \cdot 3$ & $20 \cdot 1$ & $23 \cdot 8$ & $16 \cdot 0$ & $18 \cdot 9$ & 22.5 \\
\hline
\end{tabular}

TSF, triceps skinfold thickness; BSF, biceps skinfold thickness; SIF, suprailiac skinfold thickness; SSF, subscapular skinfold thickness; R: resistance $(\Omega)$; Xc: reactance $(\Omega)$; RI, resistance index ((height (cm) $\times$ height

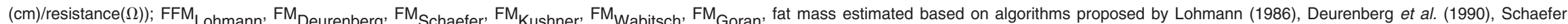

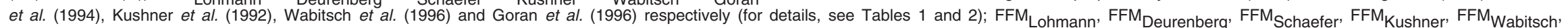

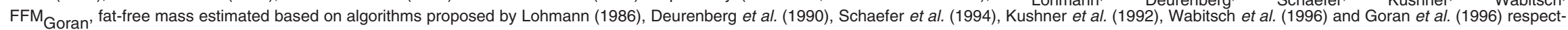
ively (for details, see Tables 1 and 2 ). 
dent variations in the estimates of \% FM: $12 \cdot 0-14.6$ for boys and 13.1-14.9 for girls according to Lohmann (1986); 8.5-10.8 for boys and $10 \cdot 1-11 \cdot 1$ for girls according to Deurenberg et al. (1990). However, when values for \% FM estimated according to these authors were compared, high values for $r(0.99)$ were obtained for both genders.

For BIA the use of different equations to estimate \% FM resulted in different median values for $\% \mathrm{FM}$, varying from 13.8 to 31.4 for boys and from 18.6 to 33.4 for girls (Table $3)$. When compared with the 90th percentile of anthropometrically-derived \% FM (according to Deurenberg et al. (1990), for example, mean cut-off point for $\% \mathrm{FM} \geq 20$ )
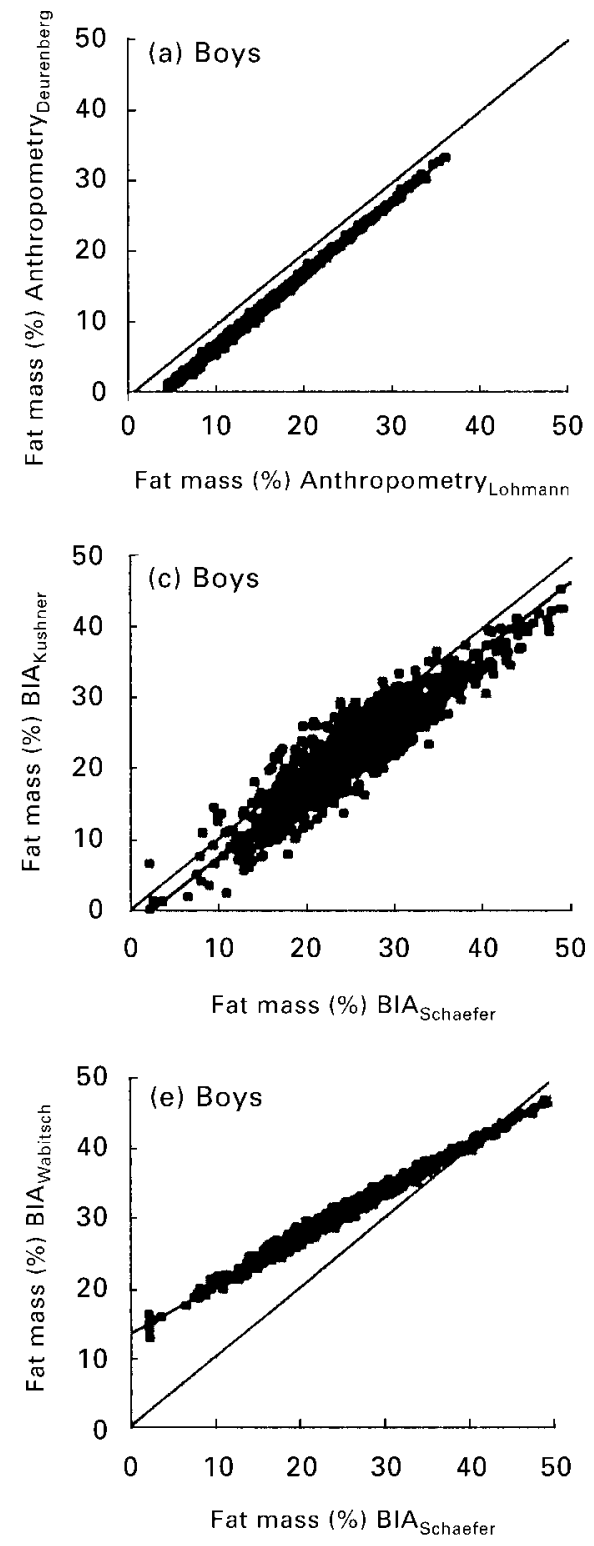

the prevalences of overweight varied between 14.7 and $42.3 \%$ (calculated according to Goran et al. 1996) and up to 98.4 and $98.5 \%$ (calculated according to Wabitsch et al. 1996) for boys and girls respectively. The influence of different algorithms was also reflected in differences in the 10th, 50th and 90th percentiles of FM and FFM on both \% and $\mathrm{kg}$ basis (Table 4).

$\%$ FM derived from anthropometry calculated according to Lohmann (1986) and Deurenberg et al. (1990) showed a high consistency in the data (Fig. 1(a and b)). However, when \% FM values derived from BIA and calculated according to the different published algorithms were compared there were some inconsistencies in the data
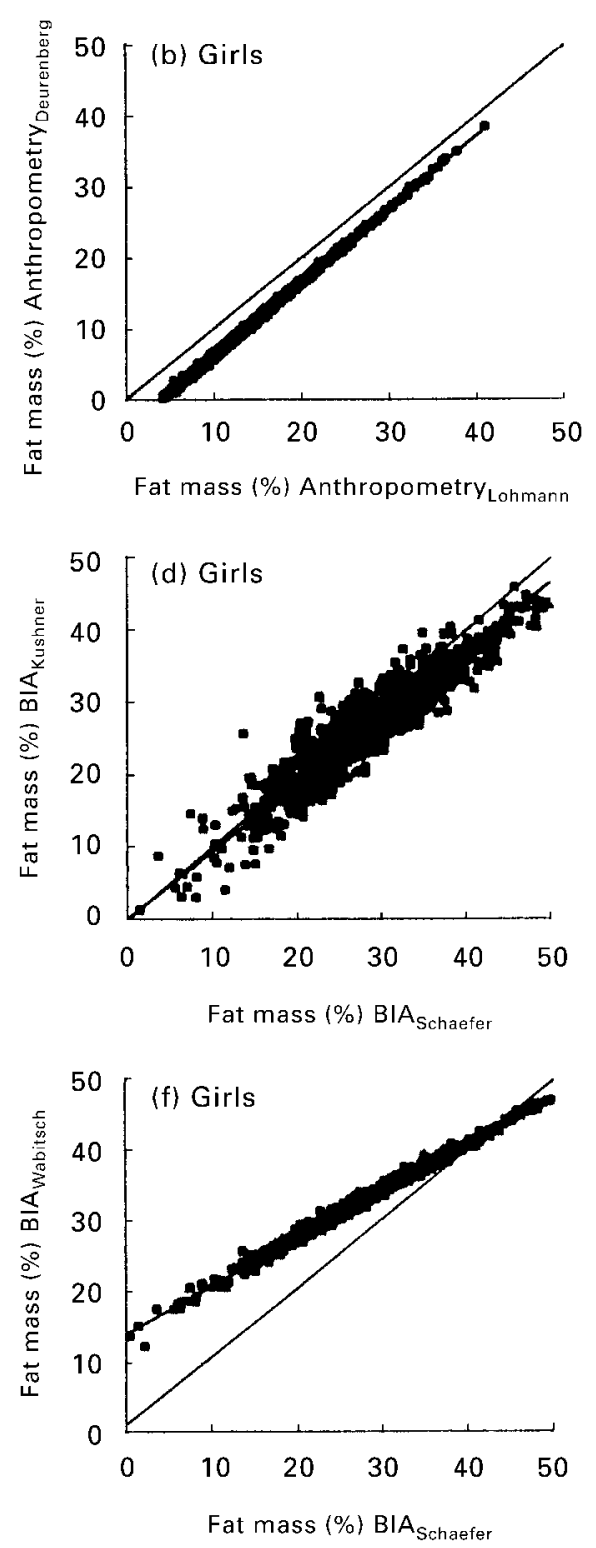

Fig. 1. Comparison of estimates of fat mass: $(a, b)$, derived from anthropometric measurements according to Lohmann (1986) and Deurenberg et al. 1990; Anthropometry Lohmann and Anthropometry Deurenberg respectively); (c-f), derived from bioelectrical impedance analysis (BIA) according to Schaefer et al. (1994), Kushner et al. (1992) and Wabitsch et al. (1996; BIA Schaefer, BIA Kushner $_{\text {and BIA }} A_{W a b i t s c h}$ respectively) in 2286 5-7-year-old boys (a,c,e) and girls (b,d,f) from Kiel, north-west Germany. For details of subjects and procedures, see p. 164 and Tables 1 and 2. Regression equations: (a) $y=1.0302 x-4.1332, R^{2} 0.9977$; (b) $y=1.0301 x-4.1464, R^{2} 0.9987$; (c) $y=0.9698 x-$ 2.2496, $R^{2} 0.8836$; (d) $y=0.9261 x+0.551, R^{2} 0.8839$; (e) $y=0.6813 x+13.51, R^{2} 0.9895 ;$ (f) $y=0.6724 x+13.982, R^{2} 0.99$. 

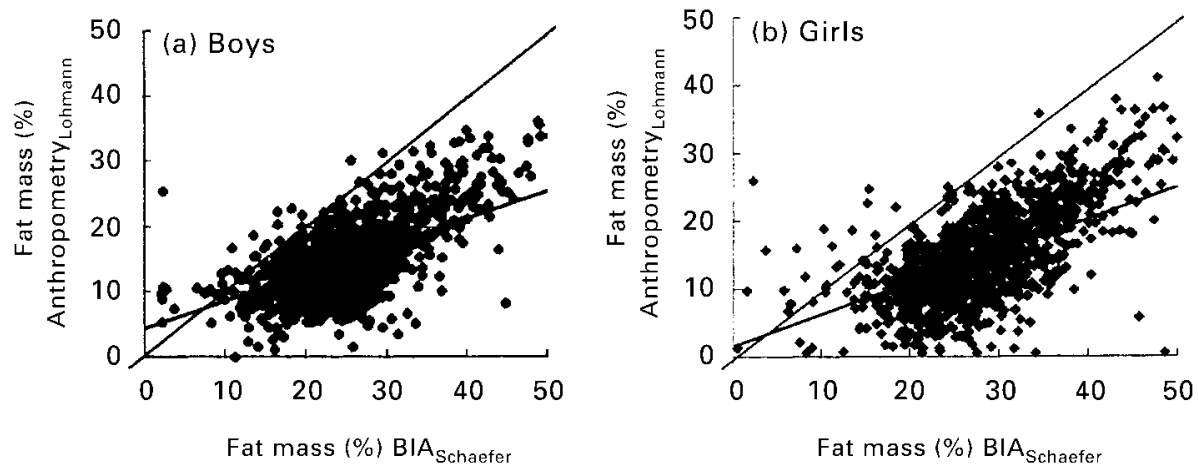

Fig. 2. Comparison of estimates of fat mass from bioelectrical impedance analysis data (data analysis according to Schaefer et al. (1994); BIA $_{\text {Schaefer }}$ ) and skinfold measurements (data analysis according to Lohmann (1986); Anthropometry Lohmann) in 2286 5-7-year-old boys (a) and girls (b) from Kiel, north-west Germany. For details of subjects and procedures, see p. 164 and Tables 1 and 2. Regression equations: (a) $y=0.4219 x+4.3424, R^{2} 0.3681$; (b) $y=0.4681 x+1.5817, R^{2} 0.3418$.

(Fig. 1(c-f)). When compared with data calculated from the algorithm of Schaefer et al. (1994), the Kushner et al. (1992) algorithm underestimated \% FM (Fig. 1(c and d)). By contrast, the algorithm proposed by Wabitsch et al. (1996) overestimated \% FM when compared with the data derived from the algorithm of Schaefer et al. (1994) at low and normal \% FM, but showed some underestimation at high \% FM (Fig. 1(e and f)). \% FM derived from BIA calculated according to Schaefer et al. (1994) correlated with \% FM derived from anthropometry according to Lohmann (1986) (Fig. 2 (a and b)), as well as with \% FM as derived from the equation of Goran et al. (1996) (Fig. 3 (a and b)). A Bland \& Altman (1986) plot showed that \% FM according to Schaefer et al. (1994) overestimated \% FM according to Lohmann (1986) at normal and high $\%$ FM. By contrast \% FM according to Schaefer et al. (1994) underestimated \% FM according to Lohmann (1986) at low \% FM (Fig. 4(a and b)). Calculating the data according to Goran et al. (1996) showed some overestimation at high \% FM and underestimation at low \% FM (Fig. 5(a and b)).

There was no correlation between skinfold measurements and resistance score (sum of four skinfolds $v$. resistance index; $r 0.13$ for both genders) as well as \% FFM from anthropometry derived according to Lohmann (1986) and resistance score (\% FFM from anthropometry derived according to Lohmann (1986) $v$. resistance index; $r$ 0.1 for both genders). By contrast there was a significant relationship between the resistance score and $\mathrm{kg}$ FFM derived from anthropometric data ( $r 0.7$ for boys and $r 0.5$ for girls).

The differences between \% FM derived from anthropometry and BIA are influenced by BMI and height as well as gender. Comparing the differences in \% FM at different BMI the closest association was found in boys and girls within the $>10$ th and $<90$ th BMI percentile (Table 5). In this group the correlation was higher for girls than for boys. The lowest correlation was for children who were below the 10th BMI percentile. The correlation was also lower in children who were above the 90th BMI percentile than in children with a normal BMI. The correlation between the two measures (anthropometry and BIA) of \% FM was higher in boys than in girls (see data for $>90$ th BMI percentile as well as $<10$ th BMI percentile, Table 5). Height also affected the association between \% FM derived from either the anthropometric or BIA data; the closest correlation was seen for boys who are $>90$ th percentile for height. Within the 10th and 90th height percentiles the correlation was higher for boys than for girls. The lowest correlation was clearly in small children (height $<10$ th percentile, Table 5).

Body proportions may also have an impact on algorithms.
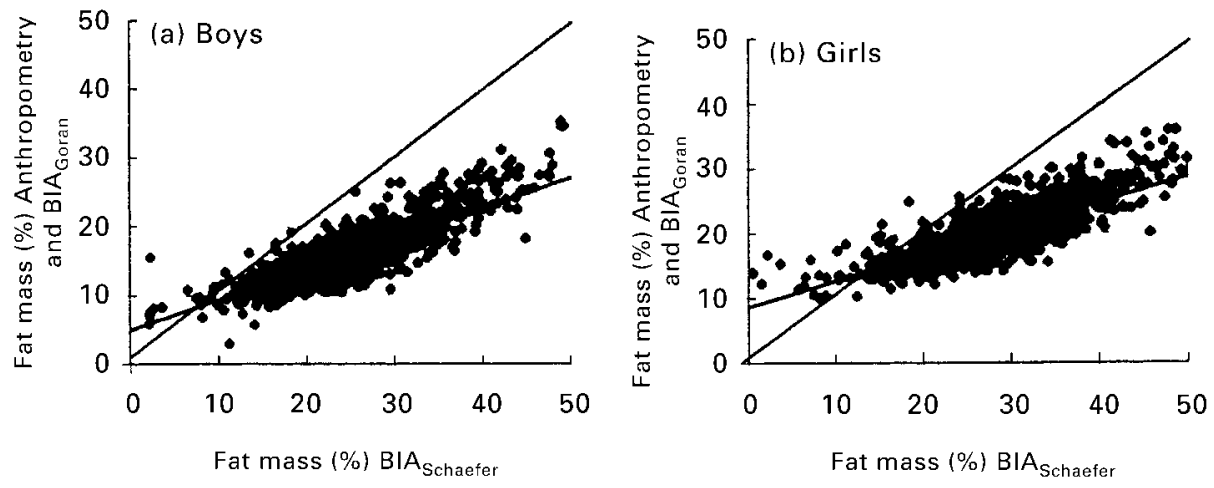

Fig. 3. Comparison of estimates of fat mass from bioelectrical impedance analysis data (data analysis according to Schaefer et al. (1994); $\mathrm{BIA}_{\text {Schaefer }}$ ) and skinfold measurements and bioelectrical impedance analysis data (data analysis according to Goran et al. (1996); Anthropometry and BIA Goran $)$ in 2286 5-7-year-old boys (a) and girls (b) from Kiel, north-west Germany. For details subjects and procedures see p. 164 and Tables 1 and 2. Regression equations: (a) $y=0.4413 x+4.9579, R^{2} 0.7218$; (b) $y=0.4024 x+8.6231 ; R^{2} 0.6512$. 

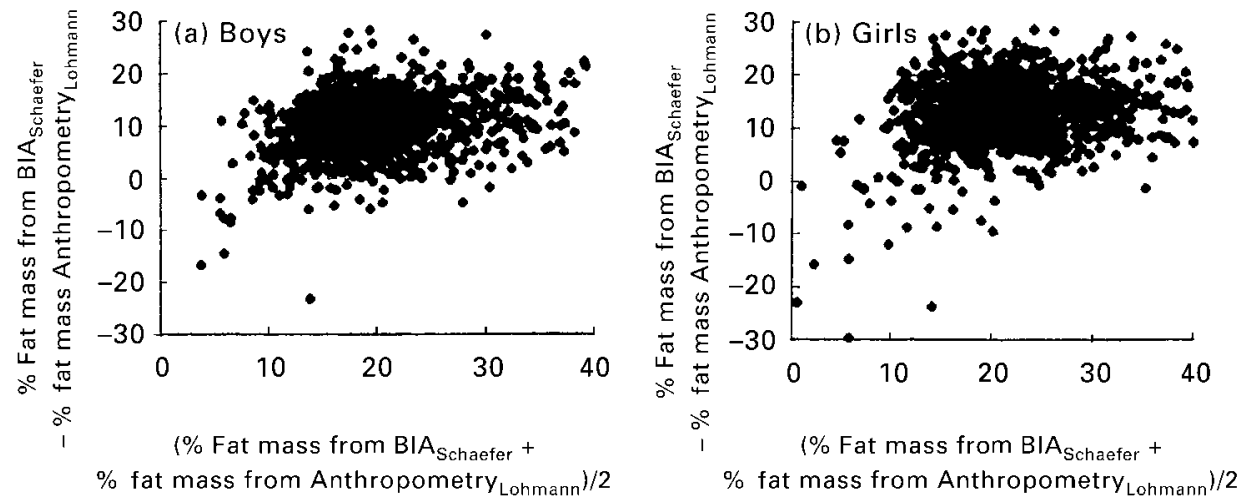

Fig. 4. Comparison of estimates of fat mass from bioelectrical impedance analysis data (data analysis according to Schaefer et al. (1994); $\mathrm{BIA}_{\text {Schaefer }}$ ) and skinfold measurements (data analysis according to Lohmann (1986); Anthropometry Lohmann) in 2286 5-7-year-old boys (a) and girls (b) from Kiel, north-west Germany in a Bland \& Altman (1986) plot. For details of subjects and procedures, see p. 164 and Tables 1 and 2 .

However, we did not find an association between resistance values and anthropometric data and lower and upper extremity length, limb circumferences and elbow breadth in a well-matched and anthropometrically characterized subgroup of twelve children (data not shown).

\section{Discussion}

BMI can be easily obtained and is generally accepted as an index of overweight and obesity in adults. However, in children and adolescents interpretation of BMI data is only possible when the age-dependent distribution is known, because BMI shows developmental variation (Hebebrand et al. 1994; Reilly et al. 1996). Several authors recommend assessment of body composition and the use of more accurate estimates of body fat as an index of overweight and obesity in children (Robinson, 1993; Schaefer et al. 1998). In field studies only simple methods can be used for body composition analysis. However, the consistency of these methods in children has not been proven in a field study.

The essential finding of the present study is that in a field study on 5-7-year-old children anthropometric and BIA measurements provide inconsistent data and result in very different estimates of \% FM within the same population. These discrepancies are in part due to the algorithms used to calculate body composition from skinfold thickness and/ or resistance. The published algorithms for estimating FM are related to the reference populations studied as well as the method used for validation (Table 2). There are considerable differences in relation to the reference populations as well as the reference methods (Table 2). Strictly speaking an algorithm is only valid for the reference population group from which it is derived. Thus, differences between the study population and the reference population affect the consistency of the results. With regard to the KOPS data, the reference population studied by Schaefer et al. (1994) is very similar to our study population. Both Schaefer et al. (1994) and Wabitsch et al. (1996) assessed German children, but Wabitsch et al. (1996) studied obese children. Although estimates of \% FM obtained by BIA as well as by skinfold measurements have been shown to be highly correlated with data obtained by more sophisticated reference methods (Table 2), the different procedures involved in the calculations result in marked differences in \% FM in our study population (Tables 3 and 4). Intra-individual comparison of the data obtained by the two techniques shows high correlation
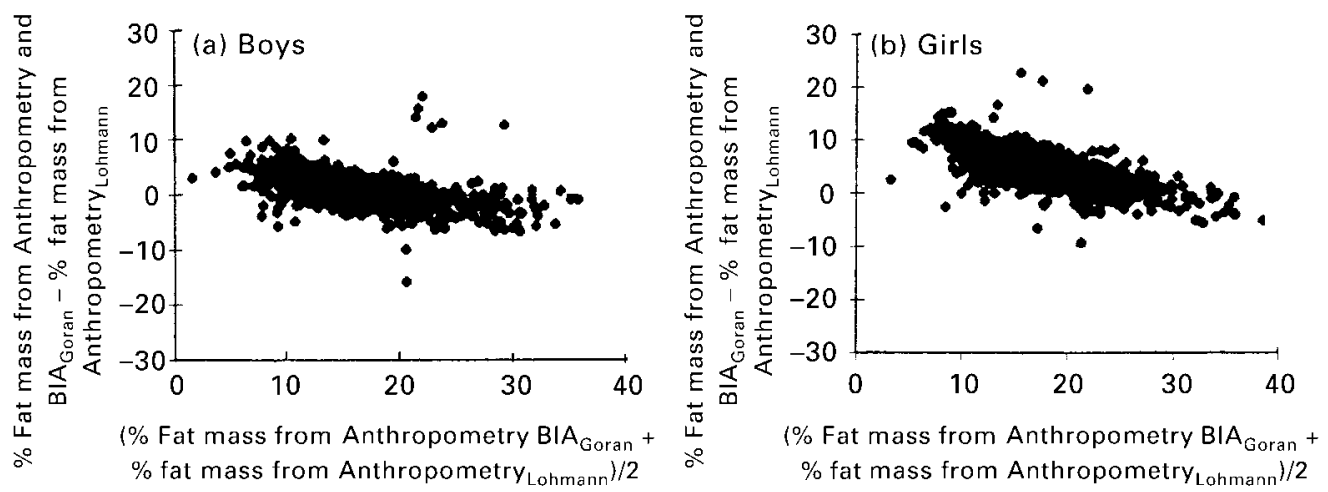

Fig. 5. Comparison of estimates of percentage fat mass from anthropometry and bioelectrical impedance analysis data (data analysis according to Goran et al. (1996); Anthropometry and BIA ${ }_{\text {Goran }}$ ) and skinfold measurements (data analysis according to Lohmann (1986); Anthropometry Lohmann) in 2286 5-7-year-old boys (a) and girls (b) from Kiel, north-west Germany in a Bland \& Altman (1986) plot. For details of subjects and procedures, see Tables 1 and 2. 
(Fig. 1(a-f)), but also some systematic over- or underestimations of \% FM in children (Figs. 2-5). For example, one boy aged 6.2 years with $7.5 \%$ FM derived from BIA according to Schaefer et al. (1994) had a corresponding value of 18.7 when derived according to Wabitsch et al. (1996). This finding points to a more general problem when comparing \% FM data from different studies using the same method but different algorithms. To compare the results of many different studies we recommend the use of an interchange table. Table 6 shows the corresponding \% FM derived from different methods and different algorithms for different percentiles of the sum of four skinfold thicknesses.

Our results agree with those of previous investigations involving smaller samples of children. Reilly et al. (1996) investigated ninety-eight healthy children to test the ability of four published equations to accurately estimate FFM from BIA in a group of prepubertal children in the UK. The authors found an overestimation, but also an underestimation, of FFM using different algorithms. Ellis (1996) investigated ninety-nine healthy children. The objective of the study was to determine the consistency of body fat measurements using four techniques for measuring body composition: BIA; bioelectrical impedance spectroscopy; total body electrical conductivity; DXA. A Bland \& Altman (1986) plot indicated differences in body fat values between methods, ranging from -0.30 (SD 6.7) $\mathrm{kg}$ to 4.2 (SD 2.7$) \mathrm{kg}$. Differences in percentage fat ranged from 0.8 (SD 3.5) to -9.9 (SD 5.2). These data suggest that classification of an individual as normal, overweight or obese was dependent on the method (Ellis, 1996). This finding is in line with our data showing that estimates of $\%$ FM in children are dependent on the method and the algorithm.

In a previous study we investigated 316 boys and 294 girls aged 5-7 years in Kiel (Mast et al. 1998). Calculating percentage fat from skinfold measurements according to Lohmann (1986) resulted in a lower estimate of \% FM ( 15.5 for boys and 13.6 for girls) than data calculated from BIA measurements (17.9 for boys and 18.6 for girls; Mast et al. 1998). The 'Chemical immaturity' and body proportions of children could explain the observed inconsistency in the estimates of \% FM. Using BIA data FM is indirectly assessed from: (1) total body water; (2) calculated FFM based on the assumption of a constant composition of FFM; (3) the difference between body weight and FFM. However, FFM does not have a constant composition in children, but shows systematic variations during development (Fomon et al. 1982; Lohmann, 1986). Malina \& Bouchard (1991) have estimated the composition of FFM during growth, and found a water content of $76.6 \%$ for boys and $77.6 \%$ for girls for children aged 5-7 years. These values exceed FFM hydration of about $73 \%$ observed in adults. It has been suggested also that there is a high inter-individual variability in FFM composition of children of a similar age (Malina \& Bouchard, 1991). Comparing \% FM calculated from BIA data based on the assumption that the water content of FFM of $76.6 \%$ (boys) or $77.6 \%$ (girls) instead of $73.2 \%$ results in higher \% FM (i.e. for boys and girls respectively the median of \% FM calculated from BIA according to Kushner et al. (1992) 
was 25.9 and 30.1 instead of 22.4 and 26.0 , while the median of \% FM from BIA according to Wabitsch et al. (1996) was 33.6 and $36 \cdot 2$ instead of 30.5 and 32.4 .

In addition to hydration of FFM, the geometric proportions of children may also differ (i.e. a greater proportion of body mass and body water is accounted for by the trunk relative to the extremities). This factor may have an impact, since the trunk only makes a minor contribution to total body impedance (National Institutes of Health, 1996). Using standard equations the body proportions of children would tend to result in an overestimation of \% FM. However, investigating several variables (i.e. lengths of arms and legs, elbow breadth and arm, waist and hip circumferences) of twelve children we did not find that different body proportions had an impact on the variance in impedance data (data not shown).

The differences between the methods of assessing \% FM are influenced by BMI as well as height. Comparing the differences in \% FM at different BMI the best correlation was found in boys and girls at normal BMI (i.e. within $>10$ th and $<90$ th BMI percentile group). In contrast, there was a poor correlation in children below the 10th BMI percentile (Table 5). Height also influences the relationship between \% FM from anthropometry and BIA data: the closest correlation was seen for boys with a height above the 90th percentile. By contrast the lowest correlation was found in short children (Table 5).

When establishing the validity of a method or an algorithm for estimating \% FM a reference method (i.e. a 'gold standard') is necessary. In general, we do not feel that we presently have a true 'gold standard' for the measurements of body composition in children. All methods used to date give method- and algorithm-specific estimates of percentage FFM and \% FM. They are based on a considerable number of assumptions. In adults densitometry or DXA have been most commonly used as reference methods. In Kiel DXA measurements in children are not permitted for ethical reasons (because of radiation exposure). In addition, densitometry as well as DXA cannot be applied in a school setting. Thus, as in many other field studies we have to use standard techniques (anthropometry and BIA) and published algorithms. Using BIA we found that the different algorithms are inappropriate in the assessment of FM in prepubertal children (e.g. Tables 3 and 4). Standard techniques as well as algorithms have to be used with caution. We feel that anthropometric as well as BIA measurements are accurate and reproducible, but at present the body composition data can only be used as method- and algorithm-specific values. Kraemer et al. (1990) have suggested five criteria for choosing a measure of adiposity. These criteria include: (a) accessibility, including characteristics such as cost, convenience, ease of use and acceptability to patients; (b) individuality, referring to independence from a population reference standard; (c) reliability, minimizing measurements error; (d) measurement validity; (e) clinical validity or the ability to predict morbidity and mortality. The present results show that BIA and skinfold measurements perform most of the criteria (a, c, d) but cannot achieve independence from a population reference standard. In the case of a population of children its clinical validity as well 
as its ability to predict morbidity and mortality are unproven. Although the measurement error can be minimized, the measurement and validity of the data remain unclear.

\section{Conclusion}

There are inconsistencies between and within anthropometric- and BIA-derived assessments of body composition in children. If anthropometry and/or BIA are used to assess FM in a field study in children we recommend caution with regard to the use of equations derived from the literature. When the results derived from different prediction formulas are compared, there are systematic over- or underestimations of $\% \mathrm{FM}$ in children. Combining BIA and anthropometric data cannot totally exclude this error. We conclude that development of a specific algorithm derived within the study population and validated against a so-called 'gold-standard' (i.e. densitometry) is preferable. If this approach is not possible, we suggest that the methods should be considered for what they directly provide (i.e. skinfold thickness and/or impedance scores). If BIA is used in field studies to obtain data from children, interpretation should make reference to an interchange table (Table 6) comparing \% FM derived using different algorithms with different percentile groups for the sum of four skinfold thicknesses.

\section{Acknowledgements}

This work was supported by grants from Deutsche Forschungsgemeinschaft $\mathrm{Mu}$ 714-5-1 und 5-2, Bonn; Verein zur Förderung der Rehabilitationsforschung in Schleswig-Holstein e.V., Lübeck; Deutsche Gesellschaft für Gesundes Leben mbH, Bickenbach; Institut Danone für Ernährung e.V., München; Wirtschaftliche Vereinigung Zucker, Bonn; Hansa Tiefkühlmenue GmbH \& Co., Hilter.

\section{References}

Bland JM \& Altman DG (1986) Statistical methods for assessing agreement between two methods of clinical measurements. Lancet i, 307-310.

Cole TJ, Bellizzi MC, Flegal KM \& Dietz WH (2000) Establishing a standard definition for child overweight and obesity worldwide: international survey. British Medical Journal 320, 1240-1243.

Cordain L, Whicker RE \& Johnson JE (1988) Body composition determination in children using BIA. Growth, Development and Aging 52, 37-40.

Deurenberg P, Pieters JJL \& Hautvast GAJ (1990) The assessment of the body fat percentage by skinfold thickness measurements in childhood and young adolescents. British Journal of Nutrition 63, 293-303.

Dietz WH (1994) Critical periods in childhood for the development of obesity. American Journal of Clinical Nutrition 59, 955-959.

Ellis KJ (1996) Measuring body fatness in children and young adults: comparison of BIA, total body electrical conductivity and DXA. International Journal of Obesity 20, 866-873.

Fomon SJ, Haschke F, Ziegler EE \& Nelson SE (1982) Body composition of reference children from birth to age 10 years. American Journal of Clinical Nutrition 35, 1169-1175.
Goran MJ, Driscol P, Johnsen R, Nagy TR \& Hunter G (1996) Cross-calibration of body composition techniques against dualenergy X-ray absorptiometry in young children. American Journal of Clinical Nutrition 63, 299-305.

Guillaume M (1999) Defining obesity in childhood: current practice. American Journal of Clinical Nutrition 70, Suppl., $126-130$.

Hebebrand J, Heseker H, Himmelmann W, Schäfer H \& Remscheid H (1994) Altersperzentilen für den Body-MassIndex aus Daten der Nationalen Verzehrsstudie einschließlich einer Übersicht zu relevanten Einflußfaktoren (Percentiles for the BMI based on Data of the German National Nutrition Study (NUS) and a Review of Relevant Factors with an Influence on Body Weight). Aktuelle Ernährungsmedizin 19, 259-265.

Houtkooper LB, Lohmann TG, Going SB \& Hall MC (1989) Validity of bioelectrical impedance for body composition assessment in children. Journal of Applied Physiology 66, 814-821.

Kraemer HC, Berkowitz RI \& Hammer LD (1990) Methodological difficulties in studies of obesity. I. Measurement issues. Annals of Behaviour Medicine 12, 112.

Kushner RF, Schoeller DA, Fjeld CR \& Danford L (1992) Is the impedance index $\left(\mathrm{ht}^{2} / \mathrm{R}\right)$ significant in predicting total body water? American Journal of Clinical Nutrition 56, 835-839.

Lohmann TG (1986) Applicability of body composition techniques and constants for children and youths. In Exercise and Sports Science Reviews, pp. 325-357 [KB Pandolph, editor]. New York: Macmillan.

Lohmann TG, Roche AF \& Martorell R (1988) Anthropometric Standardization Reference Manual. Champaign, IL: Human Kinetics Books.

Malina RM \& Bouchard C (1991) Growth, Maturation and Physical Activity. Champaign, IL: Human Kinetics Books.

Mast M, Körtzinger I, König E \& Müller MJ (1998) Gender differences in fat mass of 5-7-years old children. International Journal of Obesity 22, 878-884.

Müller MJ (1998) Ernährungsmedizinische Praxis (Clinical Nutrition). Heidelberg and Berlin: Springer.

Müller MJ, Körtzinger I, Mast M \& König E (1998) Prävention der Adipositas (Prevention of adiposity). Deutsches Ärzteblatt 95, 39-42.

Müller MJ, Mast M, Asbeck I, Langnäse K \& Grund A (2001) Prevention of obesity - is it possible? Obesity Reviews 2, $15-28$.

National Institutes of Health (1996) NIH Consensus statement. Bioelectrical impedance analysis in body composition measurement. Nutrition 12, 749-759.

Reilly JJ, Wilson F, McColl JH, Carmichael M \& Durnin VGA (1996) Ability of bioelectric impedance to predict fat-free-mass in prepubertal children. Pediatric Research 39, 176-179.

Robinson T (1993) Defining obesity in children and adolescents: Clinical approaches. Food Science and Nutrition 33, 313-320.

Rolland-Cachera MF, Cole TJ, Sempé M, Tichert J, Rossignol C \& Charraud A (1991) Body mass index variations: centiles from birth to 87 years. European Journal of Clinical Nutrition 45, 13-21.

Schaefer F, Georgi M, Wühl E \& Schärer K (1998) Body mass index and percentage FM in healthy German school children and adolescents. International Journal of Obesity 22, 461-469.

Schaefer F, Georgi M, Zieger A \& Schärer K (1994) Usefulness of bioelectric impedance and skinfold measurements in predicting fat-free mass derived from total body potassium in children. Pediatric Research 35, 617-624.

Wabitsch M, Braun U, Heinze E, Muche R, Mayer H, Teller W \& Fusch Ch (1996) Body composition in 5-18-y-old obese children and adolescents before and after weight reduction as assessed by deuterium dilution and bioelectrical impedance analysis. American Journal of Clinical Nutrition 64, 1-6. 